\title{
Comparison of visual and optical quality of monofocal versus multifocal intraocular lenses
}

European Journal of Ophthalmology $1-8$

(C) The Author(s) 2019 Article reuse guidelines: sagepub.com/journals-permissions DOl: $10.1177 / 1120672119827858$ journals.sagepub.com/home/ejo (S)AGE

\author{
Irene Altemir-Gomez',2,3, Maria S Millan', Fidel Vega ${ }^{4}$, \\ Francisco Bartol-Puyal', Galadriel Gimenez-Calvo ${ }^{2,3,5}$, \\ Jose M Larrosa ${ }^{1,2,3}$, Vicente Polo ${ }^{1,2,3}$, Luis E Pablo \\ and Elena Garcia-Martin 1,2,3
}

\begin{abstract}
Objective: To compare visual quality in patients implanted with Tecnis ${ }^{\circledR}$ monofocal (ZCB00) and multifocal (ZMB00) intraocular lenses taking into account their optical quality measured in vitro with an eye model.

Methods: In total, 122 patients participated in this study: 44 implanted with monofocal and 78 with multifocal intraocular lenses. Measurements of visual acuity and contrast sensitivity were performed. The optical quality of the intraocular lenses was evaluated in three image planes (distance, intermediate and near) using an eye model on a test bench. The metric considered was the area under the curve of the modulation transfer function.

Results: Optical quality at the far focus of the monofocal intraocular lens (area under the curve of the modulation transfer function $=66.97$ ) was considerably better than that with the multifocal lens (area under the curve of the modulation transfer function =32.54). However, no significant differences were observed between groups at the distance-corrected visual acuity. Distance-corrected near vision was better in the multifocal (0.I5 \pm 0.20 logMAR) than that in the monofocal group $(0.43 \pm 0.21 \log M A R, p<0.001)$, which correlated with the better optical quality at near reached by the multifocal intraocular lens (area under the curve of the modulation transfer function $=29 . \mathrm{I} I$ ) in comparison with the monofocal intraocular lens (area under the curve of the modulation transfer function $=5.0$ ). In intermediate vision, visual acuity was $0.28 \pm 0.16 \log M A R$ (multifocal) and $0.36 \pm 0.14 \log$ MAR (monofocal) with $p=0.014$, also in good agreement with the values measured in the optical quality (area under the curve of the modulation transfer function $=10.69$ (multifocal) and 8.86 (monofocal)). The contrast sensitivity was similar in almost all frequencies. Pelli-Robson was slightly better in the monofocal (I.73) than in the multifocal group $(1.64 ; \mathrm{p}=0.023)$. Conclusion: Patients implanted with multifocal ZMB00 achieved a distance visual acuity similar to those implanted with monofocal ZCB00, but showed significantly better intermediate and near visual acuity. A correlation was found between intraocular lenses' optical quality and patients' visual acuity. Contrast sensitivity was very similar between the multifocal and monofocal groups.
\end{abstract}

\section{Keywords \\ Intraocular lens, monofocal lens, multifocal intraocular lens, optical bench, optical quality}

Date received: 4 August 2018; accepted: II January 2019

\footnotetext{
'Department of Ophthalmology, Miguel Servet University Hospital, Zaragoza, Spain

${ }^{2}$ Aragon Health Sciences Institute (IIS Aragón), Zaragoza, Spain 3University of Zaragoza, Zaragoza, Spain

${ }^{4}$ Applied Optics and Image Processing Group (GOAPI), Faculty of Optics and Optometry of Terrassa, Universitat Politecnica de Catalunya, BarcelonaTech, Barcelona, Spain
}

${ }^{5}$ Department of Ophthalmology, Hospital Provincial de Nuestra Señora de Gracia, Zaragoza, Spain

\section{Corresponding author:}

Irene Altemir-Gomez, Department of Ophthalmology, Miguel Servet University Hospital, C/ Padre Arrupe, Consultas Externas de Oftalmología, 50009 Zaragoza, Spain.

Email: irenealtemir@gmail.com 


\section{Introduction}

Advances in cataract surgery techniques ${ }^{1}$ and improvements in optics quality of intraocular lenses (IOLs) have led to this kind of surgery attempting not only to improve patients' vision but also to provide them with good visual quality. ${ }^{2}$

The appearance of multifocal lenses made a revolutionary change in kind of this surgery, allowing spectacle independence on a daily basis. . $^{3,4}$

Multifocal lenses were designed to provide good distance, intermediate and near vision, in such a way that a fixed-focus lens cannot meet. ${ }^{5}$

We could objectively measure the performance of IOLs through imaging quality metrics (e.g. modulation transfer function-based metrics) measured using a model eye on an optical bench ${ }^{6,7}$ and study their correlation with visual performance of pseudophakic patients. By means of these correlated preclinical metrics, ${ }^{8}$ it would be possible to predict the relative change in the clinical outcomes given a change in the IOL design tested on optical bench.

In this study, the visual quality of implanted patients was tested by measuring their distance, intermediate and near visual acuity (VA) and their contrast sensitivity (CS) in photopic and mesopic conditions. VA was related to the measured optical quality of the IOLs at the corresponding image distances, being the latter estimated from the area under the curve of the modulation transfer function (AMTF) curve measured in an optical bench. The AMTF has proved to be an efficient preclinical metric to predict average VA outcomes in pseudophakic patients. ${ }^{9,10}$

\section{Methods}

This study was undertaken in Miguel Servet Hospital (Zaragoza, Spain) where 122 patients with bilateral cataracts were recruited prospectively for the study. All of them underwent cataract surgery with posterior IOL implantation in the capsular bag. The lens implanted was randomly chosen between monofocal and multifocal. All the patients enrolled in this study were informed about its nature and signed informed consent to undergo the clinical examination in accordance with the tenets of the Declaration of Helsinki. The study was approved by the hospital ethics committee.

Inclusion criteria were as follows: aged less than 75, bilateral cataracts (VA higher than 0.6 in logMAR scale), corneal astigmatism less than $1 \mathrm{D}$ and IOL power between +17 and +27D. Exclusion criteria were previous intraocular surgery, zonular-break risk factors, intraoperative problems, endophthalmitis, irregular astigmatism, deep amblyopia, systematic syndromes, glaucoma or corneal disorders.

All subjects underwent an ophthalmologic evaluation: refraction, VA assessment, slit-lamp examination, Goldmann tonometry, indirect fundoscopy, endothelial cell counting (SP-1P Topcon specular microscope).
Optical coherence biometry (IOLMaster 500 Advanced Technology V.7.3; Carl Zeiss, Jena, Germany) was performed.

All patients were operated by the same surgeon using the same technique of phacoemulsification lens with 2.2$\mathrm{mm}$ incisions. Each patient received the same type of lens in both eyes.

The postoperative revisions were performed at 1 day, 1 week, 1 month and 3 months after surgery. All patients underwent the postoperative evaluation of their VA 1 month after the surgery of their second eye. VA was presented in $\log$ MAR scale. The measurements included uncorrected distance visual acuity (UDVA), best-corrected distance visual acuity (CDVA), best distance-corrected intermediate visual acuity (DCIVA) and best distance-corrected near visual acuity (DCNVA). The VAs were measured at distance $(6 \mathrm{~m}$, vergence $+0.2 \mathrm{D})$, intermediate $(63 \mathrm{~cm}$, vergence $+1.6 \mathrm{D})$ and near $(33 \mathrm{~cm}$, vergence +3.0D) using Early Treatment Diabetic Retinopathy Study (ETDRS) charts. We recall that it is essential to set cautiously the near and intermediate distances in studies in which multifocal lenses are evaluated.

We take into account some other function besides VA; today, the most widespread and used measure in clinical treatment and investigation is CS. ${ }^{11}$ We measured it at four frequencies: 3, 6, 12 and 18 cycles/degree (cpd) in photopic and mesopic conditions with CSV-1000 test. We also measured the CS with the Pelli-Robson test, which evaluates only one special frequency $(1 \mathrm{cpd})$. This test was performed in photopic conditions with best distance correction. These measurements were performed in all the patients 3 months after surgery.

\section{IOLs}

The IOLs implanted were Tecnis ZCB00 as monofocal IOL and Tecnis ZMB00 as multifocal IOL (Figure 1(a)). ${ }^{12}$ Both IOLs are made of the same material (hydrophobic acrylic, refractive index 1.47) and share the same wavefront aspheric optics design that produces a maximum spherical aberration (SA) of $-0.27 \mu \mathrm{m}$ for a 6.0 -mm eye pupil.

The Tecnis ZMB00 is a pupil-independent, full-aperture diffractive multifocal IOL of $+4.00 \mathrm{D}$ near addition (at the IOL plane). The lens has an anterior aspheric surface and a posterior spherical one with the diffractive profile. Theoretically, about $41 \%$ of the incident light energy would be directed to the near focus, another $41 \%$ to the distance focus and the remaining energy (approximately $18 \%$ ) would be expended in higher diffraction orders. ${ }^{13}$

\section{Optical quality assessment}

Optical quality of distance, intermediate and near foci was determined with an optical test bench shown in Figure 1(b) and described in detail elsewhere. ${ }^{14}$ The setup included a model eye with an artificial cornea and is consistent with 


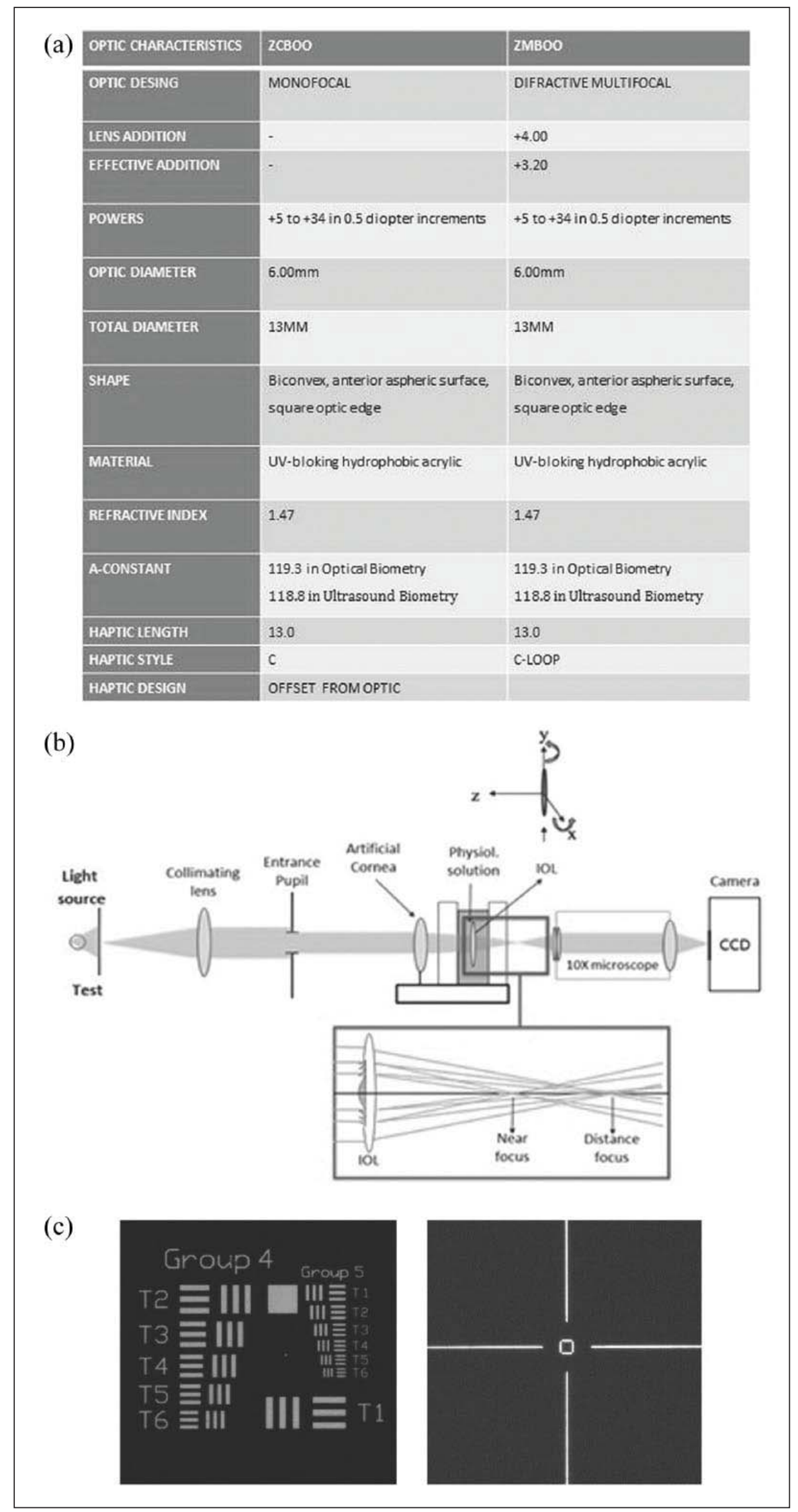

Figure I. (a) Optical characteristics of monofocal (ZCB00) and multifocal (ZMB00) intraocular lenses (IOLs); (b) model of artificial eye in optical bench; (c) representation of USAF test (a) and four-slit pattern test (b). 
Table I. Visual acuity (logMAR) and contrast sensitivity for the multifocal and monofocal IOL groups.

\begin{tabular}{|c|c|c|c|c|c|}
\hline & \multicolumn{2}{|c|}{ Monofocal IOL } & \multicolumn{2}{|c|}{ Multifocal IOL } & \multirow[t]{2}{*}{$P$} \\
\hline & Mean & SD & Mean & SD & \\
\hline UDVA photopic & 0.04 & \pm 0.11 & 0.02 & \pm 0.10 & 0.251 \\
\hline DCVA photopic & -0.02 & \pm 0.06 & -0.02 & \pm 0.08 & 0.421 \\
\hline UDVA mesopic & 0.28 & \pm 0.13 & 0.25 & \pm 0.15 & 0.284 \\
\hline CDVA mesopic & 0.22 & \pm 0.10 & 0.20 & \pm 0.12 & 0.278 \\
\hline DCIVA photopic & 0.36 & \pm 0.14 & 0.28 & \pm 0.16 & 0.014 \\
\hline DCNVA photopic & 0.43 & \pm 0.21 & 0.15 & \pm 0.20 & 0.001 \\
\hline SC Pelli-Robson & 1.73 & \pm 0.18 & 1.64 & \pm 0.21 & 0.023 \\
\hline CSVI000 3 cpd photopic & 1.67 & \pm 0.26 & 1.52 & \pm 0.25 & 0.030 \\
\hline CSVI000 6 cpd photopic & 1.80 & \pm 0.24 & 1.72 & \pm 0.23 & 0.116 \\
\hline CSVI000 I2 cpd photopic & 1.40 & \pm 0.24 & 1.36 & \pm 0.26 & 0.358 \\
\hline CSVI000 I8 cpd photopic & 0.98 & \pm 0.21 & 0.92 & \pm 0.29 & 0.235 \\
\hline CSVI000 3 cpd mesopic & $\mathrm{I} .47$ & \pm 0.34 & 1.35 & \pm 0.25 & 0.436 \\
\hline CSVI000 6 cpd mesopic & 1.54 & \pm 0.20 & 1.46 & \pm 0.34 & 0.180 \\
\hline CSVI000 I2 cpd mesopic & 0.99 & \pm 0.32 & 0.86 & \pm 0.37 & 0.059 \\
\hline CSVI000 I8 cpd mesopic & 0.48 & \pm 0.34 & 0.46 & \pm 0.33 & 0.796 \\
\hline
\end{tabular}

IOL: intraocular lens; UDVA: uncorrected distance visual acuity; DCVA: distance-corrected visual acuity; DCIVA: distance-corrected intermediate visual acuity; DCNVA: distance-corrected near visual acuity; SD: standard deviation; CS: contrast sensitivity; CSV: contrast sensitivity vision; cpd: cycles per degree; VA: visual acuity.

Bold letters indicate statistically significant differences.

the International Organization for Standardization (ISO) 11979-143 2:2014.2. ${ }^{15}$ It was taken into consideration the recommendation of using an artificial cornea affected by $\mathrm{SA}$, similar to the average human cornea. An iris diaphragm, with a variable aperture, was placed in front of the artificial cornea as the entrance pupil in order to control the size of the beam reaching the artificial cornea and thus the level of SA introduced by the model eye (without the IOL). ${ }^{16}$ To this extent, our artificial cornea provided $+0.27 \mu \mathrm{m}$ of SA for a $6.0 \mathrm{~mm}$ pupil diameter. A green light emitting diode (LED525E; Thorlabs GmbH, Munich, Germany), with emission centred at $525 \mathrm{~nm}$ and a fullwidth at half-maximum spectral bandwidth of $\pm 15 \mathrm{~nm}$, was used to illuminate either a 1951 USAF (United States Air Force) resolution test or a four-slit pattern test object for MTF measurement (Figure 1(c)).

The MTF curve was obtained in three image planes (distance, intermediate and near) from the Fourier transform of the line spread function of the slit pattern images. 6,8 The AMTF was determined by the integration of the MTF curve between 0 and 100 cycles per millimetre. The higher the AMTF values achieved, the better the optical quality of the IOL. For each IOL and focus, measurements were conducted with two pupil sizes at the IOL plane: a $3 \mathrm{~mm}$ lens aperture to compare with photopic pupils and a $4.5 \mathrm{~mm}$ aperture to compare with mesopic light conditions.

\section{Statistical analysis}

Statistical analyses were carried out with the Statistical Package for the Social Sciences (SPSS 15.0; SPSS Inc.,
Chicago, IL). Mean values and standard deviations were calculated for every parameter. It was proved that samples adjusted to normality with the Kolmogorov-Smirnov test and the Student's t-test were used accordingly.

\section{Results}

The recruited patients (122) were divided into two groups according to the IOL implanted: monofocal (44) and multifocal (78). Mean age was $60.72 \pm 8.98$ years in the monofocal group and $63.27 \pm 6.42$ years in the multifocal group. There were no differences between gender (chi-square test, $\mathrm{p}=0.337$ ) and age (Student's t-test, $\mathrm{p}=0.090)$ in both groups.

No relevant differences between IOL base powers were found: mean of $+22.75 \pm 2.16 \mathrm{D}$ (monofocal) and $+22.65 \pm 2.22 \mathrm{D}$ (multifocal) with $\mathrm{p}=0.817$.

\section{Clinical measurements}

The results of VA in different light conditions are shown in Table 1 and Figure 2(a). In photopic conditions, no statistically significant differences between UDVA or distance-corrected visual acuity (DCVA) were observed. A noticeable decrease in distance VA, both corrected (DCVA) and uncorrected (UDVA), was reported in both groups (monofocal and multifocal) in mesopic conditions. The largest differences in visual quality were found in near vision (DCNVA; $p<0.001)$. Smaller but statistically significant differences were also found in intermediate vision (DCIVA). 


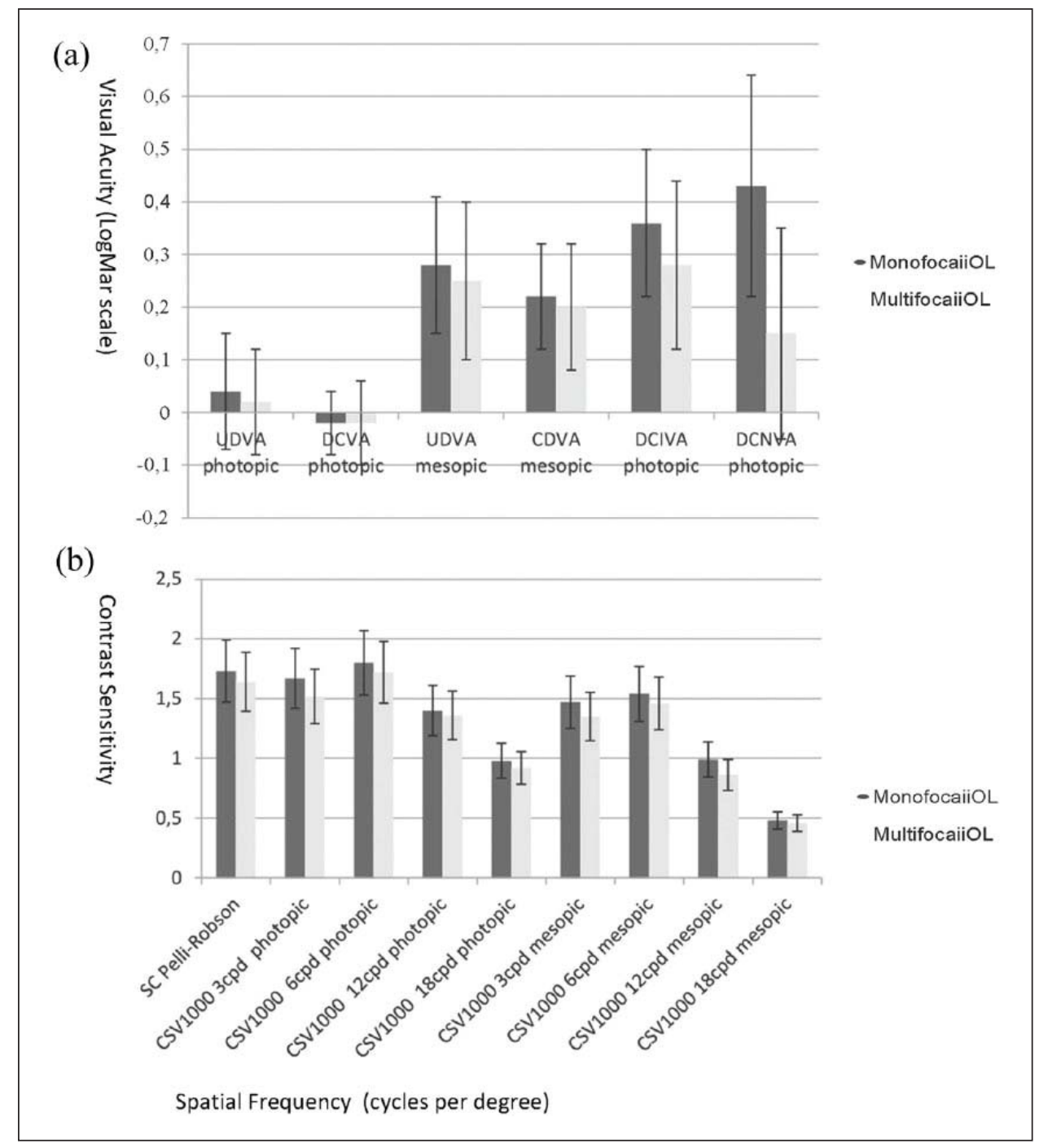

Figure 2. Bar graphs with the mean and standard deviation of (a) visual acuity (logMAR scale) and (b) contrast sensitivity for the multifocal and monofocal groups.

With regard to the postoperative CS, the monofocal group showed consistently slightly better results than the multifocal group, but these differences failed to reach the significance level except for the spatial frequency of $3 \mathrm{cpd}$ under photopic conditions and with the Pelli-Robson test (Table 1 and Figure 2(b)).

\section{Optical bench measurements}

Figure 3 shows the images of the USAF test and the MTF obtained with a pupil of $3.0 \mathrm{~mm}$ in the optical test bench with the monofocal and multifocal IOLs in three image planes: distance $(0.0 \mathrm{D})$, intermediate $(+2.0 \mathrm{D})$ and near $(+4.0 \mathrm{D})$ vergence with respect to the IOL plane. These vergences (or equivalently these image planes) are translated and referred to the glass plane. These planes approximated the planes at which VA was evaluated: $6 \mathrm{~m}(0.17 \mathrm{D})$,
$63 \mathrm{~cm}(1.6 \mathrm{D})$ and $33 \mathrm{~cm}(3.0 \mathrm{D})$, although they did not exactly match. Good-quality USAF images were obtained at distance with both lenses, although the image obtained with the monofocal lens had the best contrast. A reasonably good image at near was obtained only with the multifocal ZMB00 IOL. None of the lenses were able to produce a good image at the intermediate plane. Although the USAF images allow a fast and qualitative comparison of the optical quality of the lenses, a quantitative assessment of the differences in optical quality was carried out by evaluating the AMTF between 0 and 100 cycles per millimetre (i.e. the AMTF, which corresponds to the shaded region below the MTF curves in Figure 3). The four-slit pattern test was used in the optical bench to measure MTF in all IOLs. The AMTF values obtained with the pupils of 3.0 and $4.5 \mathrm{~mm}$ are included in Table 2 and show that in every image plane the larger the pupil, the lower the AMTF 


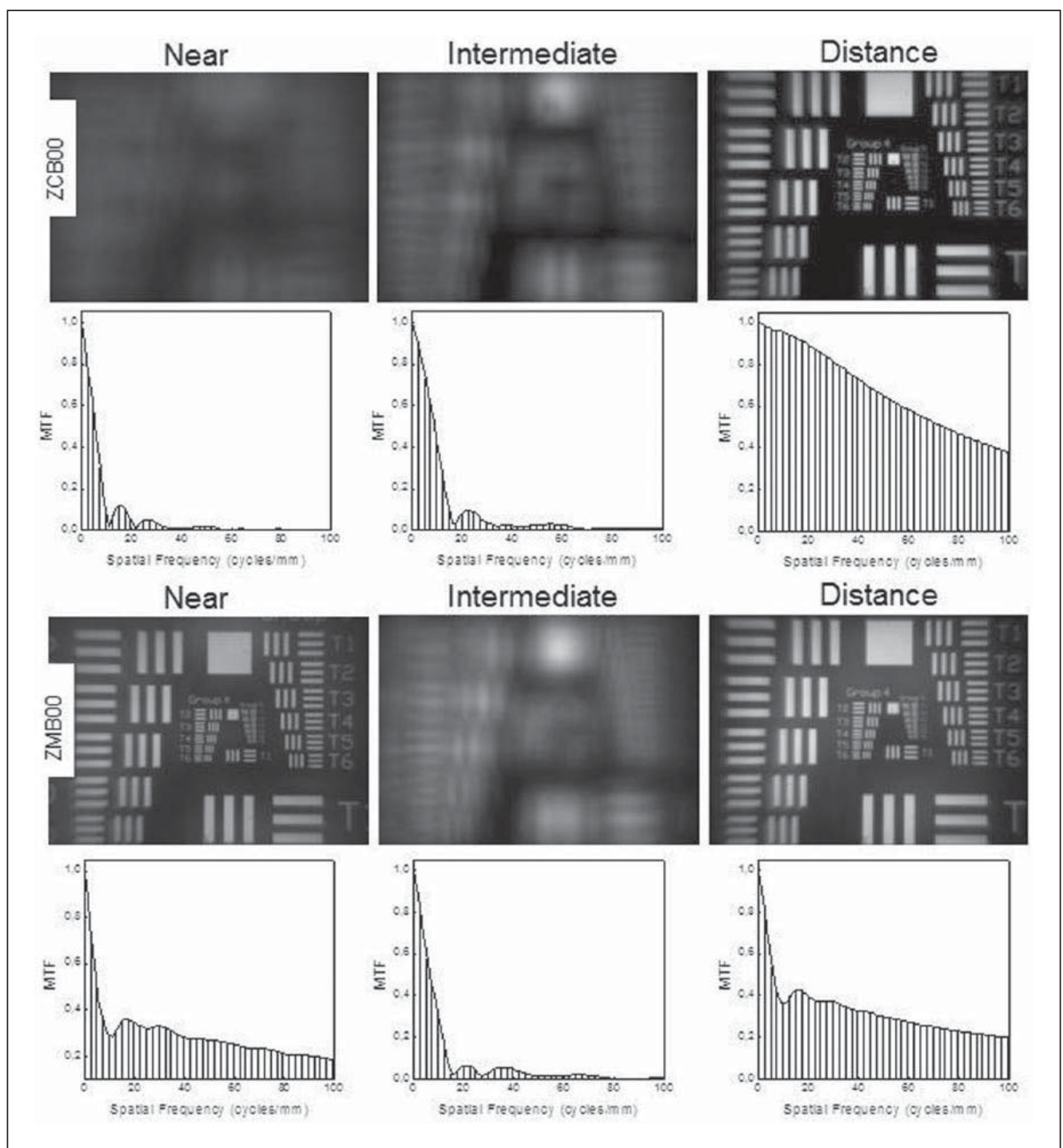

Figure 3. Images of the USAF test obtained with the monofocal ZCBOO and multifocal ZMBOO IOLs in the optical bench at the distance, intermediate and near image planes. The associated MTF at each image plane is shown below. The shadowed regions of the MTF curves are integrated, from 0 to 100 cycles $/ \mathrm{mm}$, to calculate the AMTF (AMTF values in Table 2).

for both IOLs. Moreover, the monofocal ZCB00 had the largest AMTF (and thus the best optical quality) at the distance focus, but fails at the near image plane. In contrast, the multifocal ZMB00 showed similar AMTF values (and thus similar optical quality) at the distance and near image planes. Finally, at the intermediate focus both IOLs decreased in their AMTF values.

\section{Relationship between clinical VA and laboratory AMTF}

The mean DCVA, DCIVA and DCNVA values for both IOLs were compared with their corresponding AMTF values. This is shown in Figure 4 in the case of a pupil of $3.0 \mathrm{~mm}$, showing an association between AMTF and VA in which, in general, larger values of AMTF correspond to better VA scores (lower logMAR values). However, this association can be thought to be compatible with a linear function only for relatively low values of AMTF. Thus, for AMTF values over a certain threshold (about 35), in the region shaded in grey on the right side of Figure 4, further improvement of the AMTF did not necessarily imply a significant gain in VA, and consequently the VA values remained almost constant and good (values around 0.0 $\log$ MAR) even though the AMTF value overpassed the threshold.

\section{Discussion}

In our study under photopic conditions, the values of UDVA and CDVA were similar. The UDVA results were remarkable in this study: $+0.04 \pm 0.11$ in the 
Table 2. AMTF values obtained with the monofocal ZCBO0 and multifocal ZMBO0 at each image plane with the pupils of 3.0 and $4.5 \mathrm{~mm}$.

\begin{tabular}{lcc}
\hline & ZCB00 lens & ZMB00 lens \\
\hline 3-mm pupil & & \\
Distance & 66.97 & 32.54 \\
Intermediate & 10.69 & 8.86 \\
Near & 5.0 & 29.11 \\
4.5-mm pupil & & \\
Distance & 56.95 & 28.11 \\
Intermediate & 7.15 & 6.03 \\
Near & 5.0 & 24.63 \\
\hline
\end{tabular}

AMTF: area under the curve of the modulation transfer function.

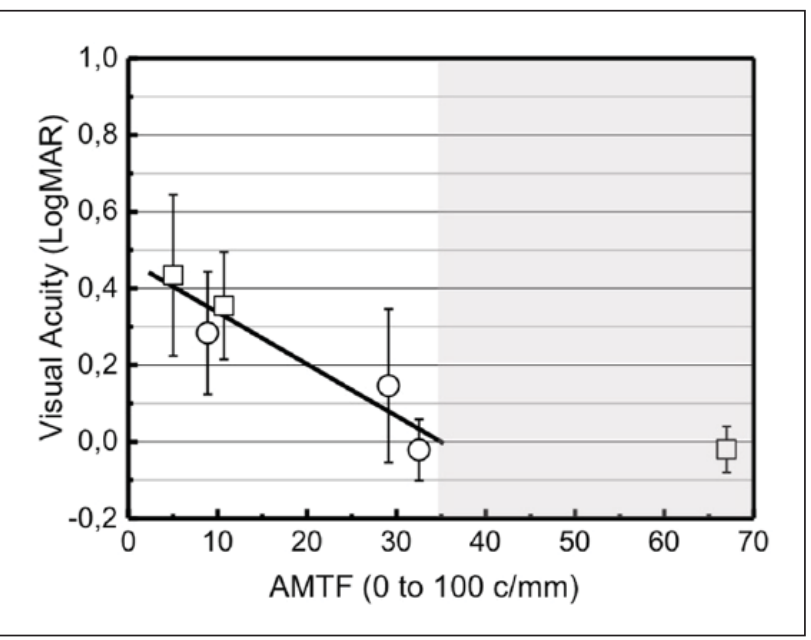

Figure 4. Distance, intermediate and near visual acuity values (mean \pm standard deviation) versus the AMTF. The squares represent the monofocal ZCBOO parameters and the circles represent the multifocal ZMB00 parameters. The solid line is a linear fit of data with AMTF values lower than 35 .

monofocal IOL group and $+0.02 \pm 0.10$ in the multifocal IOL group. With the best DCVA, the values slightly improved: $-0.02 \pm 0.06$ (monofocal) and $-0.02 \pm 0.08$ (multifocal). A reduction of the distance VA was found in both groups under mesopic light conditions. Our results are in good agreement with those obtained by Chang, ${ }^{17}$ who evaluated VA in 32 patients implanted with the multifocal ZMB00 IOL. He found the values of $-0.02 \pm 0.08$ and $-0.09 \pm 0.08$ for UDVA and CDVA, respectively, in photopic conditions. In agreement with our results, they also found a reduction of patients' VA in mesopic conditions.

Furthermore, there was a significant improvement in the near vision of the multifocal group in comparison with the monofocal one. We found the values of $+0.43 \pm 0.21$ (monofocal) and $+0.15 \pm 0.20$ (multifocal) for DCNVA. Chang ${ }^{17}$ reported better values, while Ye et al. ${ }^{18}$ found
DCNVA of $+0.025 \pm 0.11$ in patients with multifocal ZMA00 $(+4.0 \mathrm{D})$ and $+0.55 \pm 0.12$ with the monofocal ZA9003. These results confirm that the multifocal ZMB00 IOL improves the near visual function of the patients with respect to a monofocal implant.

Regarding the intermediate visual outcomes, the DCIVA worsened in both groups with regard to their own scores in the distance vision. Moreover, the intermediate vision in the monofocal group was worse than that in the multifocal one $(+0.36 \pm 0.14$ vs $+0.28 \pm 0.16 ; p=0.014$, respectively). The values of DCIVA in the multifocal group are similar or slightly worse than the results reported in previous studies with Tecnis multifocal IOLs and other diffractive multifocal IOLs. ${ }^{17,18}$ In fact, some limitations have been reported after the implantation of multifocal IOLs with high addition, which has motivated the clinical evaluation of new IOLs with lower addition. 19,20

Regarding CS, our study supports the premise that this function is not seriously compromised in the multifocal group in comparison with the monofocal one, since we only found slightly higher CS in eyes with the monofocal IOL, with the Pelli-Robson test and the low frequency of 3 cpd in the CSV-1000 test. This is consistent with the results reported in previous studies. ${ }^{21}$ Packer et al. ${ }^{22}$ compared the CS values between patients implanted with Tecnis multifocal ZM900 IOLs and monofocal IOLs and reported worse CS with Tecnis ZM900, but, similarly to our study, these differences were not clinically significant.

Finally, the relationship between VA and optical quality of the IOLs was evaluated. The largest differences of VA between the two groups are reported for near vision, in which, consistent with the bifocal design of the ZMB00 IOL, the VA results of the multifocal group were much better than those achieved by the monofocal one (DCNVA of $+0.15 \pm 0.20$ in multifocal vs $+0.43 \pm 0.21$ in monofocal; $\mathrm{p}<0.001)$. This result correlated well with the large differences in AMTF $\left(\mathrm{AMTF}_{\text {multifocal }}=29.11 \mathrm{vs}\right.$ $\mathrm{AMTF}_{\text {monofocal }}=5$ ).

In the case of distance vision, both groups had similar values of DCVA despite the fact that the AMTF of the multifocal ZMB00 was noticeably smaller than the AMTF of the monofocal ZCB00 $\left(\mathrm{AMTF}_{\text {multifocal }}=32.54 \mathrm{vs}\right.$ $\left.\mathrm{AMTF}_{\text {monofocal }}=66.97\right)$. The fact that this difference in optical quality does not imply an improvement in VA at the clinical level can be justified taking into account that once patients have reached the level of optical quality for which VA is already good (around 0.0 $\log$ MAR), additional improvements in the optical quality of the IOLs did not produce any detectable improvement in their VA. These results are in agreement with the findings of Felipe et al..$^{23}$ who showed that a decrease in the average modulation value of at least $24 \%-25 \%$ is necessary in order to cause a significant decrease in VA. ${ }^{9}$

Intermediate vision (DCIVA) worsened in both groups regarding their respective scores in distance vision. The 
AMTF reported in the intermediate image plane was very similar for both IOLs $\left(\mathrm{AMTF}_{\text {monofocal }}=10.69 \mathrm{vs}\right.$ $\mathrm{AMTF}_{\text {multifocal }}=8.86$, respectively), although the DCIVA were slightly but significantly better in the multifocal group $(+0.36 \pm 0.14$ monofocal vs $+0.28 \pm 0.16$ multifocal, with $\mathrm{p}=0.014)$.

One of the strengths of this study is the correlation of the results obtained in the laboratory in optical bench with the clinical results in patients. However, the conclusions of this study should be taken into consideration according to the limitations thereof, since the sample size is relatively small. Further prospective studies could be needed.

\section{Declaration of conflicting interests}

The author(s) declared no potential conflicts of interest with respect to the research, authorship and/or publication of this article.

\section{Funding}

The author(s) disclosed receipt of the following financial support for the research, authorship, and/or publication of this article: This study was supported by the DPI2013-43220-R and DPI201676019-R projects of the Ministry of Economy and Competitiveness and FEDER funds of Spain.

\section{References}

1. Alió JL, Abdou AA, Puente AA, et al. Femtosecond laser cataract surgery: updates on technologies and outcomes. $J$ Refract Surg 2014; 30(6): 420-427.

2. Stattin M, Zehetner C, Bechrakis NE, et al. Comparison of IOL-Master 500 vs.Lenstar LS900 concerning the calculation of target refraction: a retrospective analysis. Ophthalmologe 2015; 112(5): 444-450.

3. Alió JL, Tavolato M, DelaHoz F, et al. Near vision restoration with refractive lens exchange and pseudoaccommodating and multifocal refractive and diffractive intraocular lenses: comparative clinical study. J Cataract Refract Surg 2004; 30(12): 2494-2503.

4. Mojzis P, Pena-Garcia P, Liehneova I, et al. Outcomes of a new diffractive trifocal intraocular lens. $J$ Cataract Refract Surg 2014; 40(1): 60-69.

5. Javitt JC and Steinert RF. Cataract extraction with multifocal intraocular lens implantation: a multinational clinical trial evaluating clinical, functional, and quality-of-life outcomes. Ophthalmology 2000; 107(11): 2040-2048.

6. Goodman JW. Introduction for Fourier optics. 2nd ed. New York: McGraw-Hill, 199 pp.

7. Plaza-Puche AB, Alió JL, MacRae S, et al. Correlating optical bench performance with clinical defocus curves in varifocal and trifocal intraocular lenses. J Refract Surg 2015; 31(5): 300-307.

8. Alarcon A, Canovas C, Rosen R, et al. Preclinical metrics to predict through-focus visual acuity for pseudophakic patients. Biomed Opt Express 2016; 7(5): 1877-1888.
9. Cardona G, Vega F, Gil MA, et al. Visual acuity and image quality in five diffractive intraocular lenses. Eur $J$ Ophthalmol 2017; 28: 36-41.

10. Montes-Mico R, Lopez-Gil N, Perez-Vives C, et al. In vitro optical performance of nonrotational symmetric and refractive-diffractive aspheric multifocal intraocular lenses: impact of tilt and decentration. J Cataract Refract Surg 2012; 38(9): 1657-1663.

11. Schmickler S, Bautista CP, Goes F, et al. Clinical evaluation of a multifocal aspheric diffractive intraocular lens. $\mathrm{Br}$ J Ophthalmol 2013; 97(12): 1560-1564.

12. Yamauchi T, Tabuchi H, Takase K, et al. Comparison of visual performance of multifocal intraocular lenses with same material monofocal intraocular lenses. PLoS ONE 2013; 8(6): e68236.

13. Castignoles F, Flury M and Lepine T. Comparison of the efficiency, MTF and chromatic properties of four diffractive bifocal intraocular lens designs. Opt Express 2010; 18(5): $5245-5256$.

14. Vega F, Alba-Bueno F and Millan MS. Energy distribution between distance and near images in apodized diffractive multifocal intraocular lenses. Invest Ophthalmol Vis Sci 2011; 52(8): 5695-5701.

15. ISO 11979-2:2014. Ophthalmic implants - intraocular lenses - part 2: optical properties and test methods.

16. Vega F, Millan MS and Wells B. Spherical lens versus aspheric artificial cornea for intraocular lens testing. Opt Lett 2010; 35(10): 1539-1541.

17. Chang DH. Visual acuity and patient satisfaction at varied distances and lighting conditions after implantation of an aspheric diffractive multifocal one-piece intraocular lens. Clin Ophthalmol 2016; 10: 1471-1477.

18. Ye PP, Li X and Yao K. Visual outcome and optical quality after bilateral implantation of aspheric diffractive multifocal, aspheric monofocal and spherical monofocal intraocular lenses: a prospective comparison. Int J Ophthalmol 2013; 6(3): 300-306.

19. Alfonso JF, Fernandez-Vega L, Puchades C, et al. Intermediate visual function with different multifocal intraocular lens models. J Cataract Refract Surg 2010; 36(5): 733-739.

20. Kretz FT, Gerl M, Gerl R, et al. Clinical evaluation of a new pupil independent diffractive multifocal intraocular lens with a $+2.75 \mathrm{D}$ near addition: a European multicentre study. Br J Ophthalmol 2015; 99(12): 1655-1659.

21. Vingolo EM, Grenga P, Iacobelli L, et al. Visual acuity and contrast sensitivity: AcrySof ReSTOR apodized diffractive versus AcrySof SA60AT monofocal intraocular lenses. $J$ Cataract Refract Surg 2007; 33(7): 1244-1247.

22. Packer M, Chu YR and Waltz KL. Evaluation of the aspheric Tecnis multifocal intraocular lens: one-year results from the first cohort of the food and drug administration clinical trial. Am J Ophthalmol 2010; 149(4): 577-584.e1.

23. Felipe A, Pastor F, Artigas JM, et al. Correlation between optics quality of multifocal intraocular lenses and visual acuity: tolerance to modulation transfer function decay. $J$ Cataract Refract Surg 2010; 36(4): 557-562. 